\title{
O ENSINO DE ESTATÍSTICA POR MEIO DA PESQUISA: UMA EXPERIÊNCIA A LUZ DA MODELAGEM MATEMÁTICA
}

\author{
R. A. S. V. LIMA* e P. G. F. GONÇALVES \\ Secretaria da Educação do Estado do Ceará \\ raveniavieira@gmail.com*
}

Submetido 10/07/2015 - Aceito 12/04/2017

DOI: 10.15628/10.15628/holos.2017.3514

\section{RESUMO}

$O$ imenso quantitativo de informações veiculadas pelos meios de comunicação, aliado ao crescente uso de ferramentas estatísticas, vem exigindo dos indivíduos o domínio de conhecimentos estatísticos para compreensão e para um posicionamento crítico. Apesar de sua relevância, o ensino de Estatística vem encontrando percalços na educação básica, no que se refere, dentre outros aspectos: a formação do professor, aos materiais didáticos disponíveis e a ênfase nos aspectos quantitativos. Diante desse quadro, o presente artigo apresenta uma experiência educacional voltada para o ensino e aprendizagem de Estatística sob o aporte da Modelagem Matemática. Empreendida em duas turmas do segundo ano do ensino médio de uma escola pública de Limoeiro do Norte-CE, os dados foram coletados utilizando: a observação participante, o diário de campo e o portfólio. Desenvolver uma proposta educativa adotando como fio condutor a pesquisa, além de ter contribuído para colocar os discentes como produtores de conhecimentos, favoreceu para a adoção de uma postura crítica frente a realidade. Nesse sentido, torna-se necessário ampliar o desenvolvimento de ações educativas que apresentem a matemática como uma ferramenta para compreensão e transformação da realidade.

PALAVRAS-CHAVE: Ensino e Aprendizagem; Educação Matemática; Pesquisa de Opinião.

\section{THE TEACHING OF STATISTIC THROUGH RESEARCH: AN EXPERIENCE IN LIGHT THE MATHEMATICAL MODELING}

\begin{abstract}
The quantitative immense of information conveyed by the media, coupled with the growing use of statistical tools, has required of individuals the domain of statistical knowledge for understanding and for a critical positioning. Despite its importance, the teaching of Statistical is encountering mishaps in basic education, with regard, among other things: teacher education, the teaching materials available and the emphasis on quantitative aspects. Given this situation, this article presents a focused educational experience on teaching and learning Statistics under the contribution of Mathematical Modeling. Undertaken in two classes of
\end{abstract}

the second year of high school to a public school in Limoeiro do Norte-CE, data were collected using: participant observation, the field diary and portfolio. Develop an educational proposal adopting as a guide the research, besides having contributed to placing the students as producers of knowledge, favored to adopt a critical attitude towards reality. In this sense, it becomes necessary to expand the development of educational activities that present mathematics not only as a science purely internal purposes, but also as a tool for understanding and transforming reality.

KEYWORDS: Teaching And Learning; Mathematics Education; Survey Research. 


\title{
1 INTRODUÇÃO
}

O imenso quantitativo de informações veiculadas diariamente pelos meios de comunicação, aliado ao crescente uso de ferramentas estatísticas para simplificar e respaldar as notícias, vem exigindo dos indivíduos contemporâneos o domínio de conhecimentos estatísticos para compreensão e para um posicionamento crítico diante das informações divulgadas. Segundo os Parâmetros Curriculares Nacionais (PCN):

\begin{abstract}
A compreensão e a tomada de decisões diante de questões políticas e sociais também dependem da leitura e interpretação de informações complexas, muitas vezes contraditórias, que incluem dados estatísticos e índices divulgados pelos meios de comunicação. Ou seja, para exercer a cidadania, é necessário saber calcular, medir, raciocinar, argumentar, tratar informações estatisticamente, etc. (Brasil, 1997a, p.25).
\end{abstract}

Nesse sentido, o conhecimento estatístico torna-se uma ferramenta importante na formação dos estudantes para o exercício da cidadania. Corroborando com essa afirmativa, Andrade (2008) ressalta que a alfabetização na escola contemporânea deve ultrapassar o saber ler e escrever, mas auxiliar os discentes para que consigam a partir de informações apresentadas, principalmente através de gráficos e de levantamentos estatísticos; interpretar, analisar, questionar a validade de generalizações, tomar decisões e posicionar-se criticamente.

Conforme Martins e Pontes (2010), os objetivos centrais do ensino e aprendizagem de Estatística na escolarização básica são: o desenvolvimento da literacia estatística, que engloba a capacidade de leitura e interpretação de dados e a utilização desses na resolução de questões diversas; e o desenvolvimento da capacidade de planejamento, execução, interpretação e avaliação de investigações estatísticas.

O desenvolvimento da literacia estatística visa fornecer aos estudantes ferramentas que auxiliem na resolução e compreensão de problemas diretamente relacionados ao seu contexto ou ainda aqueles frequentemente apresentados pelos meios de comunicação que necessitam de conhecimentos e pensamentos estatísticos.

No que se refere às investigações estatísticas, Lopes (2008) discute que estas possibilitam aos estudantes aprender a interpretar os resultados e a tomar consciência das possibilidades e limitações de generalização dos dados obtidos.

Apesar da importância do conhecimento estatístico para a vida contemporânea, o ensino dessa área do conhecimento vem encontrando diversos obstáculos no âmbito da educação básica.

Segundo Bayer (2004), embora grande parte dos futuros professores considerarem o ensino de Estatística importante para a formação dos alunos, esses licenciandos afirmaram que encontrarão dificuldades para ensinar esse conteúdo, principalmente por não se considerarem preparados devido uma formação inicial inadequada.

Outra dificuldade relacionada ao tema se refere aos materiais didáticos disponíveis, em particular os livros didáticos. Segundo Alves, Busatta, Macedo, Magalhães, Santos, Silva, Tambara Junior (2008), apesar desses materiais abordarem os conteúdos da referida área, de um modo geral, há uma ênfase na resolução de exercícios a partir de aplicações diretas de expressões, sem preocupação com a reflexão dos conceitos envolvidos; ou ainda grande simplificação dos conteúdos e a aplicação desses estritamente em exercícios matemáticos (Lopes \& Moran, 1999).

Investigando a forma como o ensino de Estatística vem sendo desenvolvido em algumas escolas, Cazorla (2006) e Lopes (2008) constataram que, na maioria das vezes, é dada ênfase ao aprendizado dos procedimentos para construção de tabelas e gráficos simples e para o cálculo de médias e desvio padrão; quase sempre a partir de exemplos oferecidos pelos próprios livros 
didáticos. Desse modo, a aprendizagem de Estatística é centrada na organização e representação dos dados, em detrimento do processo de interpretação e comparação dos dados para obtenção de conclusões.

Dentre as tendências da Educação Matemática, a Modelagem Matemática surge como uma alternativa para processo de ensino e aprendizagem de Estatística, ao propor que a ação educativa inicie com a proposição de um problema de natureza prática e se desenvolva através da busca por soluções apropriadas para essa situação inicial a partir dos conhecimentos que serão ensinados.

Diante do quadro discutido até aqui, o presente artigo tem o intuito de apresentar uma experiência educacional desenvolvida por Lima (2014), voltada para o ensino e aprendizagem de Estatística, segundo os pressupostos da Modelagem Matemática.

\section{MODELAGEM MATEMÁTICA NA EDUCAÇÃO MATEMÁTICA}

Desde os tempos mais longínquos, conhecimentos que viriam a ser relacionados atualmente com a Matemática têm sido utilizados para solução de problemas numa tentativa de leitura e compreensão do mundo. Nesse contexto, a Modelagem Matemática, "[...] arte de transformar problemas da realidade em problemas matemáticos, resolvê-los e, então, interpretar suas soluções na linguagem do mundo real"; surge como algo intrínseco da natureza humana (Bassanezi, 1999, p.15).

A Modelagem Matemática enquanto processo, busca soluções capazes de explicar e interpretar o fenômeno em estudo. A essas soluções, que podem ser representada em termos matemáticos (fórmulas, diagramas, gráficos, representações geométricas, equações algébricas, tabelas, programas computacionais, e outros), dá-se o nome de modelo.

De acordo Biembengut e Hein (2000), a Modelagem enquanto processo de representação de situações reais através de modelos matemáticos pressupõe a adoção de alguns procedimentos, sintetizados nas seguintes etapas: Interação, Matematização e Modelo Matemático.

A primeira etapa é a Interação, que consiste na tomada de conhecimento da situaçãoproblema, na busca pelo máximo de informações possíveis acerca dessa situação, visando obter maior clareza em relação aos caminhos a serem tomados para elaboração do modelo. Esse estudo pode ser realizado do modo indireto, através de livros e revistas e sites especializados, dentre outros; ou direto, por meio da pesquisa de campo, por exemplo.

A etapa de Matematização é o momento no qual são identificados os fatos envolvidos, classificando as informações como relevantes ou não. Além disso, é a etapa onde há o levantamento das hipóteses, seleção das variáveis e constantes envolvidas e a análise dos recursos matemáticos disponíveis para chegar a descrição das relações em termos matemáticos.

Segundo Biembegut e Hein (2000) essa é a fase mais complexa e desafiadora, pois é nela que se dará à tradução da situação-problema para a linguagem matemática na busca pelo modelo (solução).

A última etapa, denominada Modelo Matemático, consiste na interpretação da solução e na verificação do modelo construído. Nessa etapa, quando o modelo é encontrado, ocorre uma testagem para verificar em que nível ele se aproxima da situação-problema. Nesse sentido, sua interpretação deve ser feita através de análises das implicações da solução derivada do modelo que esta sendo investigado, para então, verificar-se sua adequabilidade. Caso o modelo não atenda às necessidades que o geraram, o processo deve ser retomado na segunda etapa, mudando ou ajustando as hipóteses, as variáveis e outros. (Biembengut \& Hein, 2000). 
Empregada no âmbito escolar, a Modelagem Matemática pode constituir-se como um meio para estimular o interesse dos estudantes pelos conteúdos matemáticos, ao oportunizar o estudo desses através de pesquisas de situações-problemas de aplicação no seu cotidiano e que valorizam o senso crítico (Campos, 2007).

No que se refere ao ensino e aprendizagem de Estatística, a Modelagem Matemática torna-se um método promissor por possibilitar ao estudante "[...] vivenciar a aplicabilidade dos conteúdos estatísticos e, ao mesmo tempo, desenvolver as capacidades de pesquisar, de realizar trabalhos em grupo, de discutir, de refletir, de criticar e de comunicar suas opiniões" (Campos; Ferreira; Jacobini \& Wodewotzki, 2012, p.101).

Com o enfoque em analisar o posicionamento de membros da comunidade sobre temas inerentes ao contexto dos discentes, dentre as estratégias de pesquisa que podem se adequar ao processo de modelagem, optou-se pela Pesquisa de Opinião, que consiste num levantamento que investiga a opinião de uma população sobre um determinado tema (Crespo, 2009).

Convém ressaltar que o uso da Modelagem Matemática enquanto método de ensino e aprendizagem pressupõe a adoção de uma perspectiva problematizadora, com a criação de questionamentos e/ou problemas; e investigativa, que consiste na "[...] busca, seleção, organização e manipulação de informações e reflexão [...]", o que contribui para o desenvolvimento do interesse do aluno e aguça seu senso crítico (Barbosa, 2004, p.3).

Outro aspecto relevante para o processo educacional é que a Modelagem poderá propiciar aos alunos melhores condições para tomada de decisões, uma vez que os discentes terão a partir dos resultados obtidos "[...] uma base quantitativa que poderá contribuir para a avaliação de aspectos qualitativos e quantitativos da situação apresentada de início" (Caldeira, Malheiros \& Meyer, 2011, p.29).

\section{SUJEITOS E ASPECTOS METODOLÓGICOS}

A presente investigação teve como sujeitos da pesquisa discentes de duas turmas do 20 ano do ensino médio, em 2014, da Escola de Ensino Médio Lauro Rebouças de Oliveira, situada na cidade de Limoeiro do Norte-CE.

No que se refere aos instrumentos de coleta de dados, foram utilizados: a observação participante, o diário de campo e o portfólio.

A observação participante constitui-se como um tipo particular de observação em que o pesquisador se insere no contexto e os dados (falas, ações, gestos, expressões, entre outros) vão sendo coletados enquanto os sujeitos da pesquisa seguem sua rotina natural. $O$ termo participante é usado para enfatizar "[...] a participação (do pesquisador) com registro das observações, procurando produzir pouca ou nenhuma interferência no ambiente de estudo" (Fiorentini \& Lorenzato, 2006, p.107).

Os fatos observados foram registrados em um diário de campo. Desenvolvidas em momentos mais próximos possíveis após a imersão do pesquisador no contexto investigado, as anotações realizadas no diário de campo visaram apresentar simultaneamente, um caráter descritivo, ou seja, com enfoque na descrição dos acontecimentos, ressaltando de modo mais detalhado os fatos mais relevantes para a pesquisa; e um caráter interpretativo, apoiado nas impressões e interpretações do investigador sobre os fatos observados/vivenciados.

Em relação ao portfólio, que consiste num dossiê para armazenamento das principais atividades (entrevistas, dados tabulados, gráficos, comentários interpretativos, entre outros) desenvolvidos em sala de aula, utilizamos na presente investigação no intuito de: 
Colocar o estudante como responsável por seu processo de aprendizagem, favorecendo ao professor a análise de singularidades e peculiaridades do desenvolvimento de cada um. Com esses pressupostos, entende-se que o portfólio pode ser um instrumento capaz de dar respostas a estas expectativas: emancipação e ampliação da autonomia do estudante e diagnóstico do professor (Anastasiou \& Alves, 2007, p.111).

Desse modo, a opção por esse instrumento visou, simultaneamente, auxiliar na análise e reflexão do professor e dos alunos sobre o processo de aprendizagem; e ainda na avaliação da experiência educacional por parte do professor.

Discutiremos na seção seguinte, um recorte dos resultados obtidos com a experiência educacional empreendida.

\section{MODELAGEM EM SALA DE AULA: REFLEXÕES SOBRE A EXPERIÊNCIA EDUCACIONAL}

A experiência educacional iniciou com a explanação da proposta, ressaltando as atividades que seriam desenvolvidas, o processo avaliativo e uma introdução a Estatística, dando ênfase a algumas de suas aplicações na sociedade contemporânea.

Considerando que o emprego da Modelagem Matemática e da pesquisa apresenta cláusulas diferentes (algumas até antagônicas) das regras do contrato didático que os alunos da educação básica estão habituados, explicitar os aspectos centrais da proposta visou amenizar o surgimento de eventuais dificuldades relacionadas a compreensão inadequada do contrato didático pelos discentes. Findado esse momento inicial, foi proposto aos alunos a formação de equipes e a definição do tema e subtema a ser pesquisado.

Pautadas nas recomendações dos Parâmetros Curriculares Nacionais- PCN, as temáticas propostas para as pesquisas foram os Temas Transversais, a saber: Saúde, Ética, Pluralidade Cultural, Meio Ambiente, Orientação Sexual e Temas Locais. Segundo o referido documento, promover uma educação para o exercício da cidadania requer "[...] que questões sociais sejam apresentadas para a aprendizagem e reflexão dos alunos" (Brasil, 1997b, p.25).

Visando a delimitação das questões de estudo, cada equipe realizou uma pesquisa sobre seu tema transversal e após o debate entre seus membros, foi escolhido um subtema que os estudantes consideraram relevante para o contexto local. Apresentamos a seguir os temas e subtemas escolhidos pelas equipes:

\begin{tabular}{c|l}
\hline EQUIPE & \multicolumn{1}{c}{ TEMA E SUBTEMA } \\
\hline Equipe 1 & Saúde: alimentação saudável \\
\hline Equipe 2 & Saúde: atendimento e infraestrutura nas instituições de saúde pública \\
\hline Equipe 3 & Meio Ambiente: sustentabilidade \\
\hline Equipe 4 & Diversidade Cultural: a diversidade de ritmos \\
\hline Equipe 5 & Orientação Sexual: homofobia \\
\hline Equipe 6 & Temas Locais: poluição na escola \\
\hline Equipe 7 & Saúde: a situação da saúde pública \\
\hline Equipe 8 & Ética: o que é ser ético? \\
\hline Equipe 9 & Meio Ambiente: conhecimentos ambientais dos alunos \\
\hline Equipe 10 & Diversidade Cultural: religiões \\
\hline Equipe 11 & Orientação Sexual: pílula do dia seguinte \\
\hline Equipe 12 & Temas Locais: educação pública \\
\hline
\end{tabular}

Tabela 1: Divisão das equipes das turmas investigadas 
Delimitadas as questões de estudo de cada equipe, a proposta seguiu para a elaboração dos cronogramas de pesquisa. Estabelecer as etapas da pesquisa e seus respectivos prazos foi fundamental para mostrar a exequibilidade e nortear o trabalho dos pesquisadores.

A participação dos alunos no processo de estudo, delimitação, escolha das temáticas e cronograma de suas pesquisas, tornou-se relevante para a prática educativa, pois ao participarem "[...] das decisões sobre o que e como pesquisar, os alunos estarão mais motivados para assimilar as informações obtidas. Integrarão essas informações a seus conhecimentos e as empregarão para ampliar sua visão de mundo e, consequentemente, orientar suas ações" (Lima, 2010, p.20).

Durante o momento reservado para a elaboração das perguntas dos questionários das pesquisas, as equipes encontraram dificuldades. Encontrando percalço similar durante sua pesquisa, Medonça (2008) sugere que uma das causas desse problema está relacionada ao fato de que, de modo mais recorrente, a prática em sala de aula estimula muito mais o aluno a responder perguntas do que as elaborar.

Adentrando ao momento de Matematização, conforme as etapas propostas por Biembengut e Hein (2000), foram ministrados os seguintes conteúdos: conceitos de população e amostra, representação dos dados através de tabelas e gráficos, medidas de tendência central (média, mediana e moda) e algumas medidas de dispersão (amplitude, desvio padrão e variância).

Em seguida, cada grupo determinou a população, convencionada entre as equipes como o conjunto de todos os alunos da escola; e a amostra, que todas as equipes optaram pela amostragem aleatória simples.

Antecedendo o momento de coleta dos dados, foi realizada novamente uma discussão acerca dos procedimentos para a realização da Pesquisa de Opinião. Essa retomada se deu em virtude das dúvidas dos discentes em relação a abordagem e desenvolvimento das entrevistas. Assim, reservou-se um momento da aula para discussão dos cuidados iniciais e abordagem do informante, postura correta diante do informante, desenvolvimento da entrevista, encerramento da entrevista e outros. Isso foi importante porque apesar das entrevistas terem sido feitas na própria escola, os estudantes não conheciam todos os entrevistados.

De posse dos dados, as equipes seguiram para a organização, análise, interpretação e representação dos dados. Como nem todos os grupos conseguiram entrevistar a quantidade de pessoas previamente estabelecidas para composição da amostra, foi dado um novo prazo para a conclusão. Independente de concluídas ou não, todas as equipes iniciaram a tabulação dos dados coletados no caderno.

Para organização dos dados, as equipes utilizaram os conceitos de frequência absoluta, frequência absoluta acumulada, frequência relativa e frequência relativa acumulada. Uma das situações mais recorrentes no processo de utilização desses conceitos foi o emprego inadequado do conceito das frequências (absoluta e relativa) acumuladas. Indagando 60 alunos com a pergunta "Com relação ao casamento gay, qual sua posição?", uma das equipes tabula os dados coletados da seguinte forma:

\begin{tabular}{|c|c|c|c|c|}
\hline Resposta & Frequência absoluta & $\begin{array}{c}\text { Frequência absoluta } \\
\text { acumulada }\end{array}$ & Frequência relativa & $\begin{array}{c}\text { Frequência relativa } \\
\text { acumulada }\end{array}$ \\
\hline CONTRA & 5 & 5 & 0,08 & 0,08 \\
\hline À FAVOR & 52 & 57 & 0,88 & 0,96 \\
\hline NÃO SEI & 2 & 59 & 0,04 & 1 \\
\hline
\end{tabular}

Tabela 2: Quadro construído pela Equipe 5 
Como se pode notar, tendo em vista que a variável inerente a questão acima é qualitativa nominal (isto é, está relacionada a uma qualidade ou atributo, não é numérica e não possui uma ordenação subjetiva), não faz sentido tabular os dados empregando as frequências acumuladas. Isso mostra que os alunos não compreenderam e não souberam aplicar corretamente os conceitos de frequência absoluta acumulada e frequência relativa acumulada. Outro equívoco cometido pela equipe responsável pela questão acima foi durante o cálculo da aproximação de casas decimais e das porcentagens.

Após a tabulação dos dados, as equipes realizaram as construções de gráficos para representação dos dados, no intuito de facilitar a interpretação e discussão dos mesmos. De um modo geral, as equipes não apresentaram muitas variações acerca dos tipos de gráficos. Foram usados: histogramas, gráficos de setor e de barras.

No que se refere ao processo de elaboração, constatamos que $70 \%$ das equipes construíram adequadamente os gráficos. Os erros mais comuns entre as equipes que se equivocaram durante esse momento foi na escolha apropriada do tipo de gráfico os dados que visavam representar.

Apesar dos erros na elaboração dos gráficos, a maior dificuldade das equipes foi na interpretação dos dados. Na maioria delas, a conclusão foi baseada apenas na alternativa que apresentou maior índice, sem apresentar nenhuma discussão acerca das demais alternativas que também alcançaram percentuais significativos. A título de ilustração, apresentamos a seguir o gráfico elaborado por uma das equipes:

Médicos de outros países irão resolver os problemas na saúde pública?

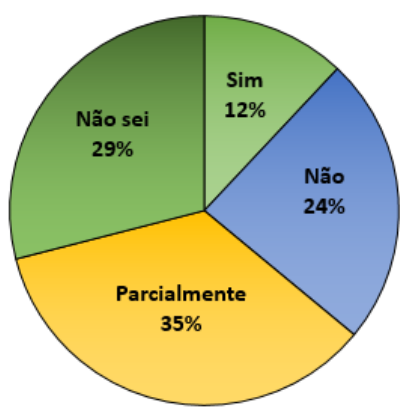

CONCLUSÃO: De acordo com a maioria dos entrevistados, a vinda de médicos estrangeiros irá resolver parcialmente os problemas da saúde pública no nosso país.

Figura 1: Gráfico e interpretação elaborada pela Equipe 2

É possível notar que apesar de terem obtidos percentuais muito próximos na frequência de algumas respostas, a equipe se deteve a apresentar a conclusão baseada unicamente na resposta de maior recorrência. Uma conclusão como essa, não expõe, por exemplo, que a opinião da população pesquisada em relação ao tema está relativamente dividida, devido essa temática ser polêmica ou pouco clara a esses indivíduos.

A etapa de validação dos modelos construídos consistiu no retorno a situação inicial e na análise da adequação do modelo em relação ao problema inicial de pesquisa. Caso o modelo se mostrasse inadequado à situação, seria necessário reformulá-lo. Entre os modelos construídos, apenas duas equipes tiveram que refazer algumas tabelas e gráficos, principalmente por equívocos cometidos no momento de tabulação dos dados. 
No momento de discussão, os alunos apresentaram os dados obtidos e as conclusões das pesquisas realizadas. Dentre as estratégias de apresentação, $85 \%$ das equipes utilizaram-se de cartazes para apresentação da pesquisa, enquanto as demais equipes relataram as informações obtidas apenas de modo oral. Apesar da simplicidade nas estratégias de explanação das pesquisas, esse momento de discussão foi bastante produtivo, ao ocasionar a participação da grande maioria dos alunos.

\section{CONSIDERAÇÕES FINAIS}

Surgindo como algo intrínseco a atividade humana, a Modelagem Matemática se constituiu como um importante método para obtenção de modelos (soluções) de problemas diversos.

A adaptação desse processo ao ensino e aprendizagem de Matemática na educação básica, torna-se promissora, sobretudo, por possibilitar uma postura ativa dos alunos enquanto pesquisadores e pela adoção de uma abordagem da matemática como ferramenta para resolução de problemas do dia a dia desses estudantes.

Adotando a Modelagem Matemática enquanto método de ensino, o presente estudo discutiu um caminho para o ensino e aprendizagem de Estatística no âmbito ensino médio.

Diante do crescente quantitativo de informações que vem apresentando instrumentos estatísticos como forma de simplificação dos dados, desenvolver uma proposta de ensino e aprendizagem de Estatística tendo como fio condutor a pesquisa sobre temas transversais a realidade dos estudantes, além de ter contribuído para colocar os discentes como produtores de conhecimentos; ao delegar a esses a tarefa de escolha do tema, planejamento, coleta, organização, análise e interpretação dos dados; favoreceu para a adoção de uma postura crítica, ao oportunizar a esses sujeitos debaterem, criticarem e questionarem sobre situações que envolviam seu contexto.

Outro aspecto relevante da pesquisa foi que a vivência em atividades de investigação estatística também mostrou aos estudantes a responsabilidade do pesquisador em relação a apresentação dos seus resultados, pois qualquer erro nas etapas da pesquisa poderia comprometer os dados, invalidando-os ou apresentando-os de forma tendenciosa.

Apesar da vivencia enquanto pesquisadores de temas de sua realidade, os estudantes encontraram dificuldades, sobretudo, na aplicação de alguns conceitos e no processo de interpretação dos dados. Um dos fatores que pode ter contribuído para a ocorrência dessas dificuldades consistiu na inexperiência dos estudantes com o desenvolvimento de pesquisas.

O estudo de conhecimentos estatísticos numa perspectiva investigativa possibilitou aos alunos colocar-se como sujeitos críticos frente a problemas provenientes de seu contexto. Desse modo, torna-se necessário ampliar o debate e o desenvolvimento de experiências educativas que apresentem a matemática como uma ferramenta para compreensão e transformação da realidade.

\section{REFERÊNCIAS}

Anastasiou, L. das G. C., \& Alves, L. P. (Orgs.). (2007). Processos de Ensinagem na Universidade: Pressupostos para as estratégias de trabalho em aula (7a ed.). Joinville: UNIVILLE.

Andrade, M. M. (2008). Ensino e aprendizagem de Estatística por meio da modelagem matemática. Dissertação de mestrado, Universidade Estadual Paulista, Rio Claro, SP, Brasil. 
Barbosa, J. C. (2004). Modelagem Matemática: O que é? Por que? Como? Veritati, Salvador, 4, 73-80.

Bassanezi, R.C. (1999). Modelagem matemática: Uma disciplina emergente nos programas de formação de professores. Congresso Nacional de Matemática Aplicada e Computacional, Santos, SP, Brasil, 22.

Bayer, A. (2004). Formandos em Matemática x Estatística na Escola: Estamos Preparados? Simpósio Sulbrasileiro de Ensino de Ciências, Canoas, RS, Brasil, 12.

Biembengut, M. S., \& Hein, N. (2000). Modelagem Matemática no Ensino. São Paulo: Contexto.

Brasil. (1997a). Parâmetros Curriculares Nacionais: Matemática. Brasília: Ministério da Educação, Secretaria de Educação Fundamental.

- (1997b). Parâmetros Curriculares Nacionais: apresentação dos temas transversais. Brasília: Ministério da Educação, Secretaria de Educação Fundamental.

Campos, C. R., Ferreira, D. H. L., Jacobini, O. R., \& Wodewotzki, M. L. L. (2012). Modelagem matemática como instrumento de interação entre aprendizagem curricular e reflexões críticas na sala de aula de estatística. Augusto Guzzo Revista Acadêmica, São Paulo, 10, 96112.

Campos, C. R. (2007). Educação Estatística: uma investigação acerca dos aspectos relevantes à didática da Estatística em cursos de graduação. Tese de Doutorado, Universidade Estadual Paulista, Rio Claro, SP, Brasil.

Cazorla, I. M. (2006). O Ensino de Estatística no Brasil. Recuperado em 07 de outubro de 2015, de http://www. sbem.com.br/gt_12/arquivos/cazorla.htm.

Crespo, A. A. (2009). Estatística fácil (19a ed.). São Paulo: Saraiva.

Fiorentini, D., \& Lorenzato, S. (2006). Investigação em educação matemática: percursos teóricos e metodológicos. Campinas: Autores Associados.

Lima, A. L. D. (2010). Nossa escola pesquisa sua opinião: manual do professor (3a ed.). São Paulo: Global.

Lima, R. A. S. V. (2014). Pesquisa de opinião e modelagem matemática: uma proposta para o ensino-aprendizagem de estatística no ensino médio. Monografia de Graduação, Universidade Estadual do Ceará, Limoeiro do Norte, CE, Brasil.

Lopes, C. A. E., \& Moran, R. C. C. P. (1999). A Estatística e a Probabilidade através das atividades propostas em alguns livros didáticos brasileiros recomendados para o Ensino Fundamental. Conferência Internacional experiências e perspectivas do ensino da estatística. Florianópolis, SC, Brasil.

Lopes, C. E. (2008). O Ensino da Estatística e da Probabilidade na Educação Básica e a Formação dos Professores. Caderno Cedes, 28 (74), 57-73.

Alves, F. N., Busatta, M. R., Macedo, C. de S., Magalhães, M. N., Santos, B. H. dos, Silva, J. M. da, Tambara Junior, S. (2008). Presença da Estatística nos ensinos fundamental e médio. Simpósio de Iniciação Científica e Pós-Graduação do IME-USP, São Paulo, SP, Brasil.

Mendonça, L. O. (2008). A Educação Estatística em um Ambiente de Modelagem Matemática no Ensino Médio. Dissertação de Mestrado, Universidade Cruzeiro do Sul, São Paulo, Brasil.

Caldeira, A. D., Malheiros, A. P. dos S., \& Meyer, J. F. da C. de A. (2011). Modelagem em Educação Matemática. Belo Horizonte: Autêntica.

Martins, M. E. G., \& Ponte, J. P. (2011). Organização e Tratamento de Dados. Lisboa: Mistério da Educação, Directório-Geral de Inovação e Desenvolvimento Curricular. 